\title{
High-performance DIRC detector for use in an Electron-Ion Collider
}

\author{
Lee Allison ${ }^{a * j}$, T. Cao ${ }^{b}$, R. Dzhygadlo ${ }^{c}$, T. Horn ${ }^{d}$, C. Hyde ${ }^{a}$, Y. Illieva ${ }^{b}$, G. Kalicy ${ }^{a d}$, P.

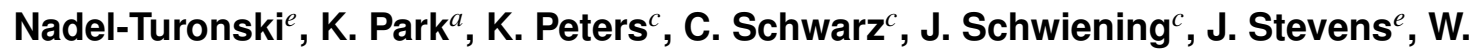 \\ $\mathbf{X i}^{e}$, and C. Zorn ${ }^{e}$ \\ ${ }^{a}$ Old Dominion University, Norfolk VA \\ ${ }^{b}$ University of South Carolina, Columbia SC \\ ${ }^{c}$ GSI Helmholtzzentrum für Schwerionenforschung GmbH, Darmstadt Germany \\ ${ }^{d}$ The Catholic University of America, Washington D.C. \\ ${ }^{e}$ Thomas Jefferson National Accelerator Facility, Newport News VA \\ E-mail: salli008@odu.edu
}

\begin{abstract}
The proposed Electron-Ion Collider (EIC) will explore the physical regime of the most fundamental particles, quarks and gluons, and their interactions, which create $95 \%$ of the mass of the visible matter in the universe. To analyze these collisions a sophisticated particle ID system must be built. A detector based on Detection of Internally Reflected Chereknov light (DIRC) technology is an attractive and radially compact solution for charged particle identification $(e / \pi, \pi / K$, $K / p$ ) at an EIC in the barrel region not accessible by traditional RICH detectors. This article will describe the status of the design and R\&D effort for a high performance DIRC envisioned for an EIC detector. The current design of the DIRC at EIC will use a new, innovative compound lens to reach remarkable resolution sufficient to extend the momentum coverage well beyond stateof-the-art, up to $6 \mathrm{GeV} / \mathrm{c}$. Performance of a prototype of the compound lens on both test bench studies as well as in a CERN test beam will also be discussed.
\end{abstract}

38th International Conference on High Energy Physics

3-10 August 2016

Chicago, USA

\footnotetext{
* Speaker.

${ }^{\dagger}$ This work was carried out with support from the U.S. Department of Energy, the BNL "Generic Detector R\&D Program for the EIC" (eRD14), and GSI Helmholtzzentrum für Schwerionenforschung GmbH.
} 


\section{The Electron-Ion Collider}

The Electron-Ion Collider (EIC) is proposed as the next major project for nuclear physics research in the United States. It will be the first accelerator capable of colliding polarized electrons with light polarized ions or heavier, unpolarized ions up to uranium. The current design of the Jefferson Lab full-acceptance central detector for the first interaction region is shown in Figure 1. This detector is divided into three (3) regions, each with a Cherenkov-based particle identification (PID) solution for pion/kaon separation: the electron end-cap which will use a modular aerogel Ring Imaging Cherenkov (RICH) detector for separation up to $10 \mathrm{GeV} / \mathrm{c}$, a hadronic end-cap which will use a dual-radiator RICH for separation up to $50 \mathrm{GeV} / \mathrm{c}$, and a barrel region using a Detection of Internally Reflected Cherenkov light (DIRC) detector for separation up to $6 \mathrm{GeV} / \mathrm{c}$. While the DIRC is not capable of the same degree of momentum separation as the aerogel or dual-radiator RICHs, it will also be largely unnecessary for PID of momentum larger than $6 \mathrm{GeV} / \mathrm{c}$ in the barrel region due to kinematic restraints. It also sports a very radially compact footprint, extending only a few $\mathrm{cm}$, making it a very attractive solution for PID in this region. More detailed information about the physics goals of EIC experiments, as well as the designs of the JLab and BNL designs for the accelerator can be found in [1], [2], and [3].

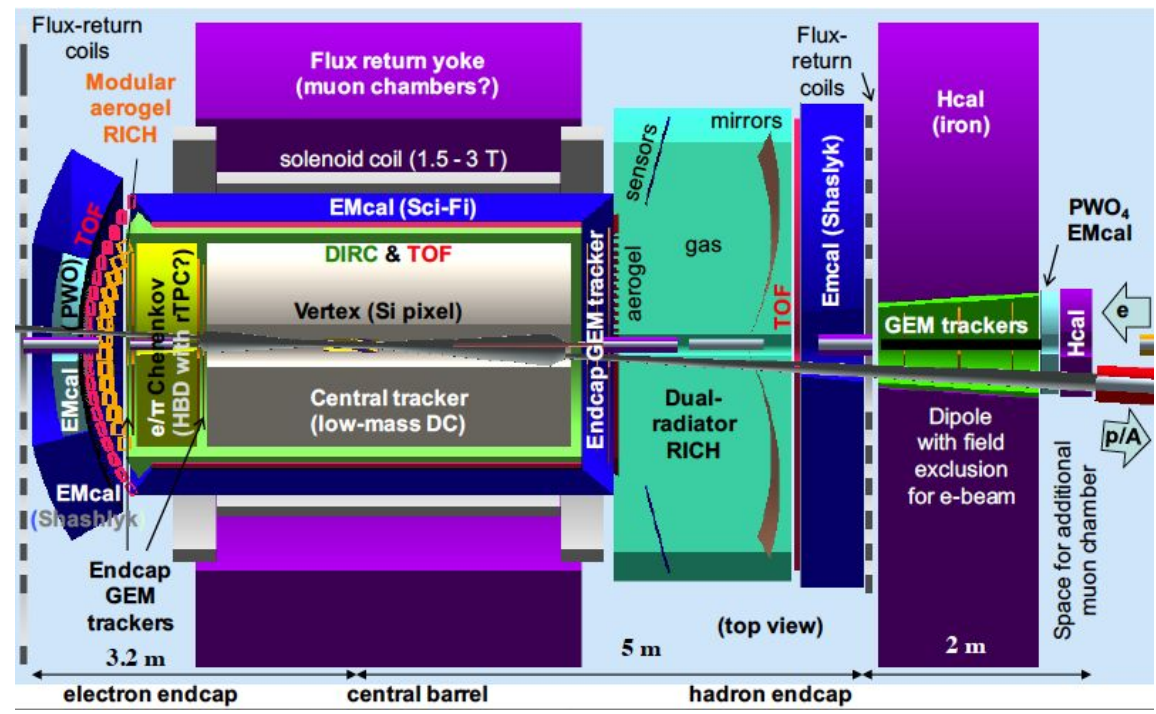

Figure 1: Top view of the current layout of the full acceptance JLab EIC detector with all subsystems. The detector is divided in to the three (3) regions: an electron end-cap to the left, a hadronic end-cap to the right, and a barrel region in the middle.

The work presented in this article was done in collaboration with the PANDA Cherenkov Collaboration, who are concurrently working on a barrel DIRC for the PANDA experiment at the FAIR facility [4]. The design for the PANDA DIRC was inspired by the BaBar DIRC at SLAC, which was the first and still only operational DIRC detector used in a full experiment and was in operation for nearly a decade [5]. The excellent performance achieved by the BaBar detector meets the requirements for the PANDA experiment, however because of the limited space the PANDA detector has been designed to be more compact with a smaller expansion volume. This smaller design requires both smaller pixels on the readout photosensors as well as a focusing optic to 
preserve the desired performance. The spacial requirements for the PANDA Barrel DIRC are very similar to those for the DIRC at EIC and therefore strong collaboration between the two groups has been invaluable.

This article describes the current design of the high-performance DIRC system envisioned for the EIC detector. The general aspects of DIRC detectors along with a discussion of required and achievable performance are discussed in section 2. The key component of the design of the highperformance DIRC detector is a special 3-layer lens, described in section 3 along with a discussion of the full detector design for the EIC. Tests of a prototype of the 3-layer lens have been conducted and setup and results are shown in section 4.

\section{DIRC Detectors}

As with other Cherenkov-based detectors, DIRC detectors take advantage of the Cherenkov effect whereby a charged particle traveling through a medium faster than the speed of light through that medium will emit photons on a cone with an opening half-angle $\theta_{C}$, called the Cherenkov angle, defined by $\cos \theta_{C}=1 / \beta n(\lambda)$, where $\beta=v / c$ is the particle's velocity, and $n(\lambda)$ is the refractive index of the medium as a function of the wavelength $(\lambda)$ of the Cherenkov photons. In the case of a DIRC the radiating medium is also used as a light guide as some of the Cherenkov photons undergo total internal reflection inside the radiator and are guided towards one end of the radiator to a readout (Figure 2a). The radiator of choice is a solid bar made of fused silica, with an index of refraction $n=1.473$. A rectangular cross section and highly smoothed and polished sides ensure that the magnitude of the Cherenkov angle is preserved during internal reflection. Photons that are created propagating away from the readout are reflected back towards the readout by a mirror. Once the photons exit the radiator they are allowed to separate through an expansion volume before being imaged in both $(x, y)$ position as well as time. The arrival position and propagation time of each detected photon are combined with tracking information to reconstruct the Cherenkov angle and determine the corresponding PID likelihoods.

a)

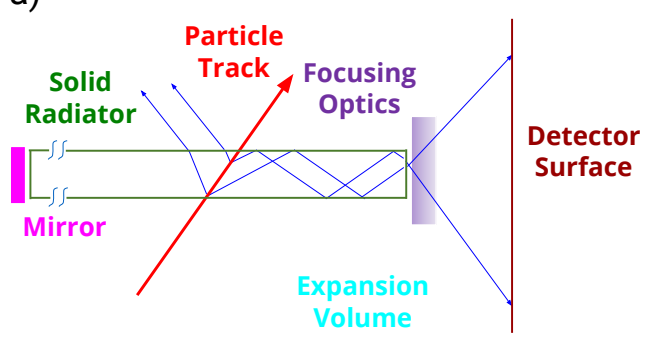

b)

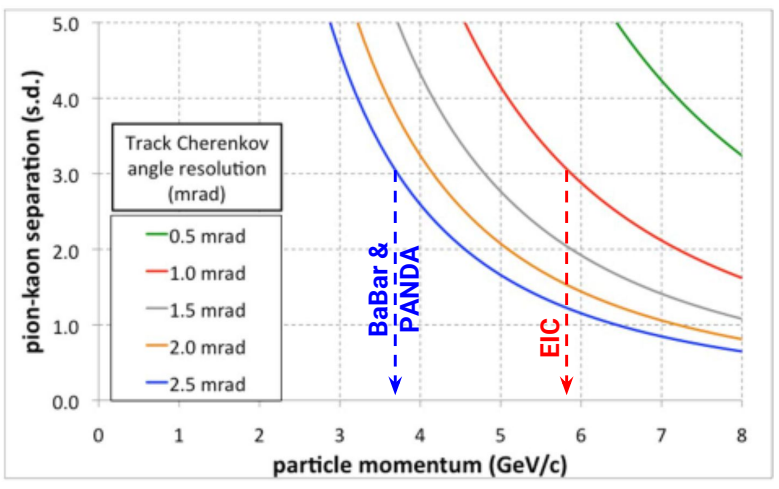

Figure 2: Components and working principle of a DIRC detector (a), and the $\pi / K$ separation in units of standard deviation as a function of momentum (b). The colored lines indicate the Cherenkov angle resolution per track, which is a combination of the DIRC detector and tracking system contributions.

There are three contributing parameters that define the track Cherenkov angle resolution $\sigma_{C}$, and thus the PID performance: the number of photons $N_{\gamma}$, the Cherenkov angle resolution per 
photon $\sigma_{C, \gamma}$, and the uncertainty of the particle direction in detector $\sigma_{\text {track }}$. Figure $2 \mathrm{~b}$ shows a plot of $\pi / K$ separation as a function of particle momentum for various assumptions of the track Cherenkov angle resolution (colored lines) as well as performance of the BaBar DIRC (blue dashed line) and the desired performance of the and PANDA and EIC DIRCs (blue and red dashed line). As can clearly be seen, EIC wishes to push the PID performance to nearly $6 \mathrm{GeV} / \mathrm{c}$ particle momentum with $3 \sigma$ separation, compared to BaBar and PANDA's $3.8 \mathrm{GeV} / \mathrm{c}$ at $3 \sigma$.

\section{High-performance DIRC Design}

The current baseline design for the barrel DIRC at an EIC (Figure 3) is $1 \mathrm{~m}$ in radius. There are 16 bar boxes, each containing $114.2 \mathrm{~m}$ long fused silica bars with a cross section of $17 \times 35 \mathrm{~mm}^{2}$. Attached to the end of each radiator bar is a mirror for redirecting photons towards the readout. For each bar box there is a fused silica prism expansion volume with a tilt angle of $38^{\circ}$ and a volume of $28 \times 39 \times 30 \mathrm{~cm}^{3}$. The detector plane of each prism is covered with $27,6902 \times 2 \mathrm{~mm}^{2}$ pixels giving a total of about 443,040 channels for recording the position and arrival time of the Cherenkov photons. The key component of this design is a new 3-layer spherical lens design that will allow to shape the focal plane in such a way that is will contour more closely to the detector plane than a traditional lens would. This allowS for better focusing and thus higher performance.
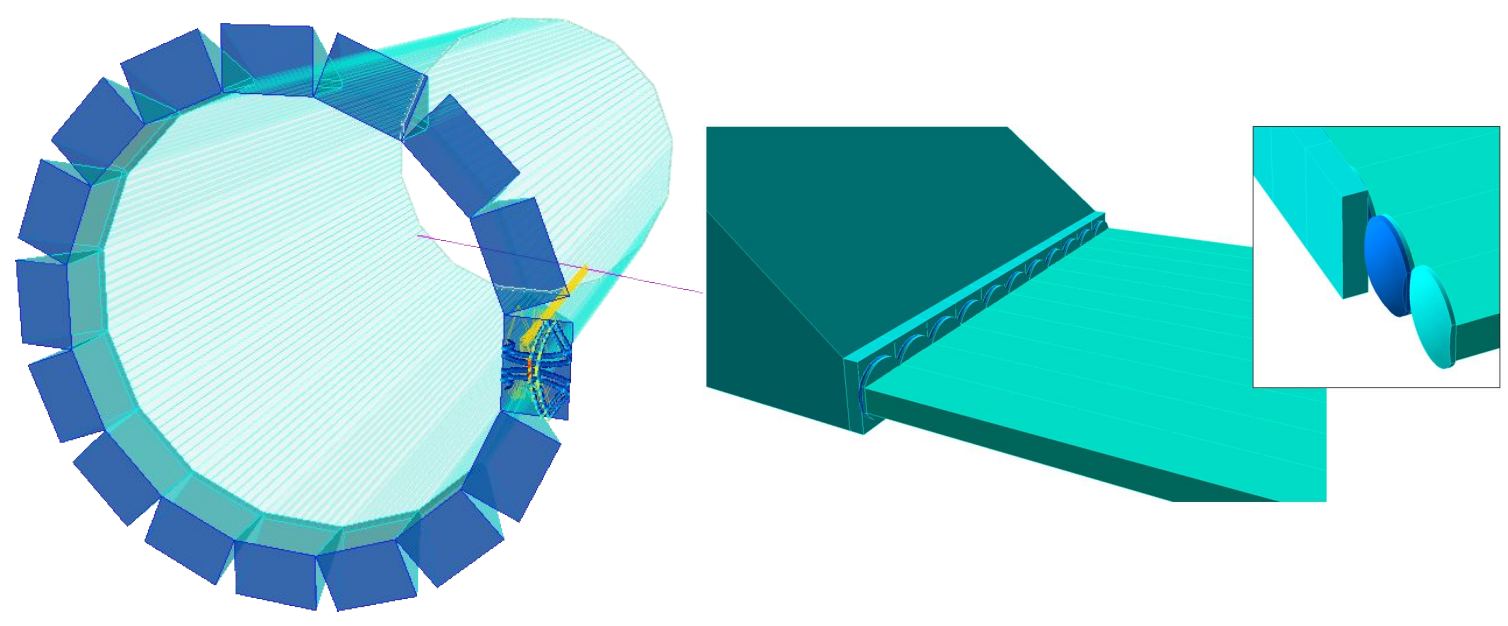

Figure 3: On the left is a full Geant4 simulation of the current baseline design of the barrel DIRC for an EIC. On the right is a zoomed in view of 11 bars attached to one expansion volume as well as the different layers of the 3-layer lens.

The lens is made of two layers of fused silica sandwiching a layer of NLaK33 glass ( $n=1.786$ at $\lambda=380 \mathrm{~nm}$ ). Two different radii of curvature create a defocusing and focusing effect which allows for shaping of the focal plane. Figure 4 shows a comparison of the design and simulated focal planes of the 2-layer lens solution (with just one curved surface) currently used by PANDA (a) and the new 3-layer lens design for EIC (b). Clearly the focal plane of the 2-layer lens is very curved and will not focus onto the detector plane for most photon angles. The 3-layer lens, however, has a focal plane that is nearly flat, which will provide a higher resolution than the 2-layer lens. 

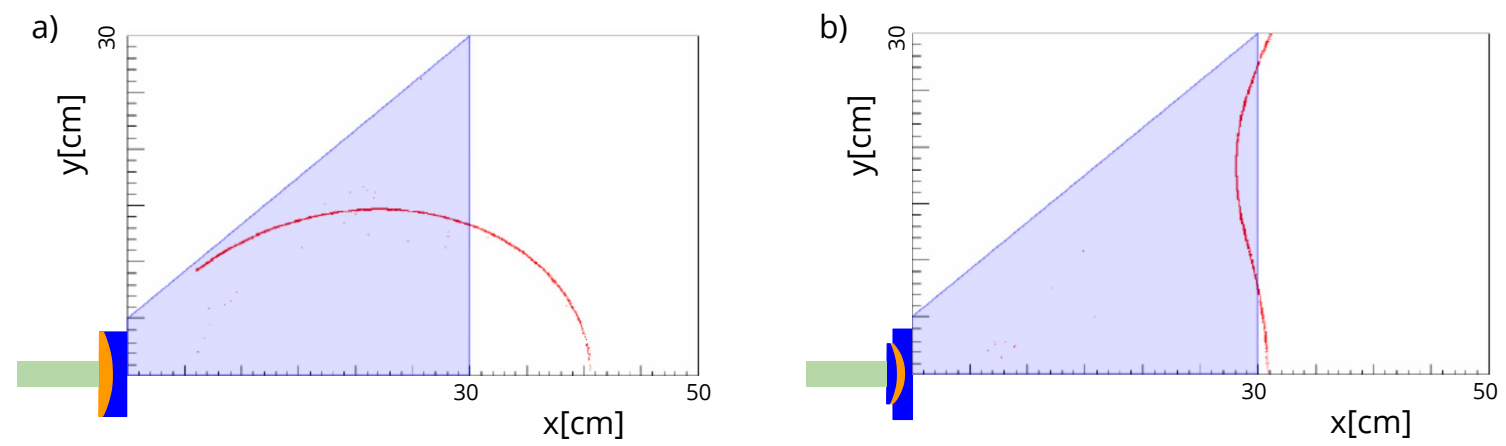

Figure 4: Drawings of bar/lens/prism combination with a 2-layer lens (a) and the new 3-layer lens design (b), along with the simulated focal planes of each lens. For each lens the dark blue color is fused silica, while the orange is NLaK33 glass.

\section{Experimental Tests of Prototype 3-Layer Lens}

The properties of this 3-layer lens design must be verified experimentally, including the shape of the focal plane, the radiation hardness of the NLaK33 material, and the lens' performance in a DIRC prototype in a particle beam. Figure 5a shows the prototype lens used for testing.
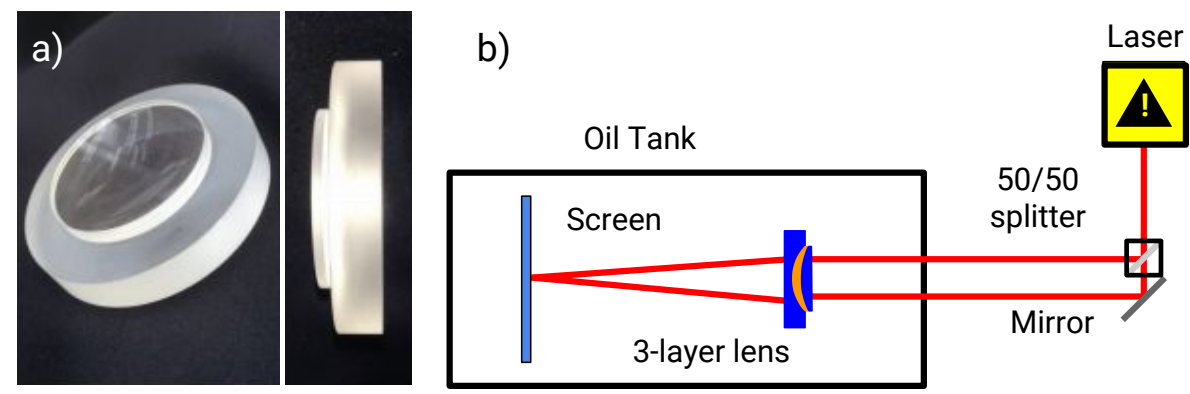

Figure 5: Top and side views of a prototype of the 3-layer lens (a), and a schematic of the optical setup used to map the focal plane of the 3-layer lens (b).

To measure the shape of the focal plane a setup has been designed and built at Old Dominion University (ODU), a schematic of which is shown in Figure 5b. A laser shines through a 50/50 beam splitter and a mirror to create two parallel, very close beams that then go through an oil tank filled with mineral oil with a similar index of refraction to that of fused silica. The beams then pass through the prototype lens and are focused onto a plastic screen that is allowed to move towards or away from the lens to find the focal point. A special holder was designed such that the lens is allowed to be rotated in two planes: in the plane of the page and into and out of the page with respect to the figure above. This allows for a full 3D mapping of the focal plane. Measurements and analysis of this measurement are still ongoing.

The measurements of the radiation hardness of the NLaK33 material will be done at The Catholic University of America (CUA) with a $160 \mathrm{keV}$ X-ray source in steps of 0.01-0.5 krad until a total of $10 \mathrm{krad}$ has been delivered to both a prototype lens and a pure sample of NLaK33. After each step the transmission of both the lens and sample will be measured with a monochromator with a reproducibility of $0.2 \%$. Alternative materials for the second layer of the lens are also being 
explored, such as lead fluoride $\left(\mathrm{PbF}_{2}\right)$ which has a high refractive index $(n=1.77)$, has been proven to be radiation hard, and has a wavelength cutoff of $300 \mathrm{~nm}$.

Because not all of the components of the high-performance DIRC baseline design for EIC are currently available (e.g. small pixel sensors) it is necessary to validate the simulation package currently used to design and optimize the system. In 2015 the PANDA Barrel DIRC group along with Lee Allison and Dr. Grzegorz Kalicy from ODU conducted a test beam at CERN with a prototype DIRC for the PANDA experiment. This was used as an opportunity to evaluate the performance of the 3-layer lens in a real particle beam. The beam was a tunable hadron beam with momentum from $1-7 \mathrm{GeV} / \mathrm{c}$. The EIC DIRC simulation package was modified to use this new geometry setup so that data could be compared to simulation. The two most important quantities measured during this test beam were the photon yield per track and the single photon resolution. Measurements were taken for a wide range of angles between the particle beam and the radiator bar (called the polar angle) and the Cherenkov reconstruction variables were extracted. Analysis of the beam data and error evaluation are still ongoing, but preliminary results are shown in Figure 6. Recall that these results are indicative of the performance of the 3-layer lens in the PANDA DIRC prototype geometry and are not reflective of the overall performance of a DIRC at EIC.

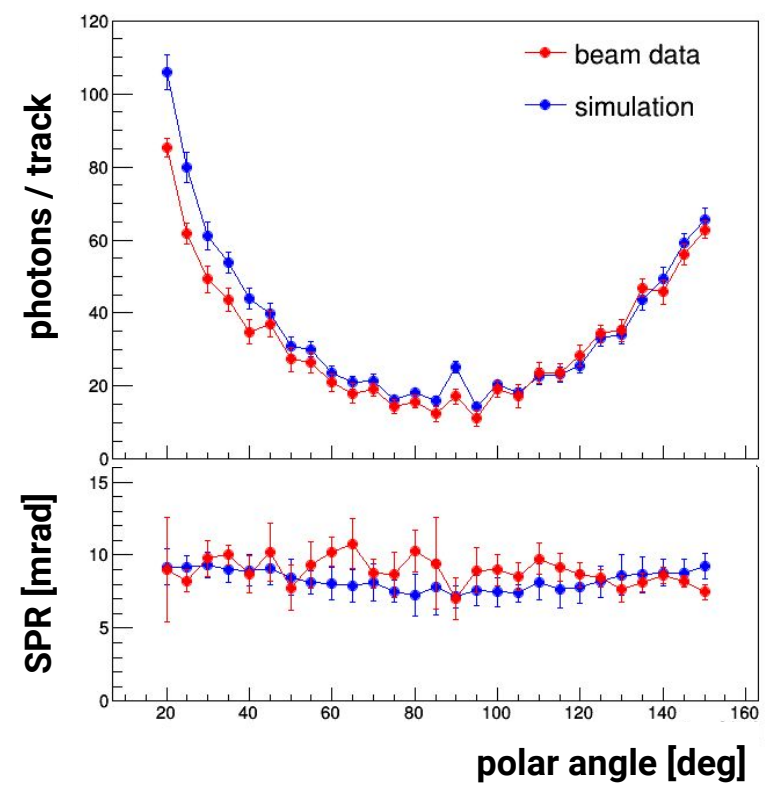

Figure 6: Preliminary results from the 2015 CERN test beam with the PANDA Barrel DIRC group. Photon yield (top) and single photon resolution (bottom) are plotted against polar angle from $20^{\circ}$ to $150^{\circ}$.

\section{Summary}

Due to it's compact design, reproducibility of results, and adequate reach in momentum, a DIRC detector is a very attractive option for the barrel region of an EIC detector. A new 3-layer lens has been designed in order to provide better focusing power to push the DIRC at EIC design to the leading edge of performance. The focal plane of the lens will be mapped out at ODU, the radiation hardness of the center NLaK33 layer will be measured at CUA, and the performance of the lens was tested in a CERN test beam to validate the Geant4 simulation package used for the DIRC at EIC. 


\section{References}

[1] A. Accardi et al., Eur. Phys. J. A52 (2016) 268, 1212.1701.

[2] S. Abeyratne et al., (2015), 1504.07961.

[3] E.C. Aschenauer et al., (2014), 1409.1633.

[4] R. Dzhygadlo et al., JINST 11 (2016) C05013.

[5] BaBar DIRC, I. Adam et al., Nucl. Instrum. Meth. A538 (2005) 281. 